\title{
Geometry of the Universe and Its Relation to Entropy and Information
}

\author{
Ioannis Haranas ${ }^{1}$ and Ioannis Gkigkitzis ${ }^{2}$ \\ ${ }^{1}$ Department of Physics and Astronomy, York University, 4700 Keele Street, Toronto, ON, Canada M3J 1P3 \\ ${ }^{2}$ Department of Mathematics, East Carolina University, 124 Austin Building, East Fifth Street, Greenville, \\ NC 27858-4353, USA
}

Correspondence should be addressed to Ioannis Haranas; yiannis.haranas@gmail.com

Received 13 June 2013; Revised 3 August 2013; Accepted 5 August 2013

Academic Editor: Gary Wegner

Copyright (c) 2013 I. Haranas and I. Gkigkitzis. This is an open access article distributed under the Creative Commons Attribution License, which permits unrestricted use, distribution, and reproduction in any medium, provided the original work is properly cited.

In an effort to investigate a possible relation between geometry and information, we establish a relation of the Ricci scalar in the Robertson-Walker metric of the cosmological Friedmann model to the number of information $N$ and entropy $S$. This is with the help of a previously derived result that relates the Hubble parameter to the number of information $N$. We find that the Ricci scalar has a dependence which is inversely proportional to the number of information $N$ and entropy $S$. Similarly, a nonzero number of information would imply a finite Ricci scalar, and therefore space time will unfold. Finally, using the maximum number of information existing in the universe, we obtain a numerical value for the Ricci scalar to be $O\left(10^{-52}\right) \mathrm{m}^{-2}$.

\section{Introduction}

Any physical system can register information just because it exists. Those systems that evolve dynamically in time not only transform but also process the information. In relation to the laws of physics, we say that these laws determine the amount of information that a given system can register (i.e., number of bits or nats) as well as the number of elementary logic operations that the given system can perform (i.e., numbers of operations). In his original paper, Landauer [1] made the statement that information is physical, and then he went on stating that: first, information is registered and processed by physical systems, and second, all physical systems register and process information. Physical systems can be described in terms of information, but information processing is related to the system description by physical laws.

In relation to the universe in a recent paper by Haranas and Gkigkitzis [2,3], the authors investigate the Bekenstein [4] bound of information number $N$ and its relation to cosmological parameters in a universe with and without cosmological constant, and they also derive a relation that describes the dependence of Hubble parameter on the amount of information $N$. The authors derive that Hubble's parameter at any time $t$ in the history of the universe can be written in the following way [3]:

$$
H(t)=\sqrt{\frac{\pi}{\ln 2}}\left(\frac{c}{\ell_{P} \sqrt{N(t)}}\right)=\frac{A}{t_{p} \sqrt{N(t)}},
$$

where $A=(\pi / \ln 2)^{1 / 2}, \ell_{P}=\sqrt{\hbar G / c^{3}}=1.616 \times 10^{-35} \mathrm{~m}, t_{p}=$ $\sqrt{c^{5} / G \hbar}=5.39 \times 10^{-44} \mathrm{~s}$ is the Planck length and Planck time, $c$ is the speed of light, and $N$ is the number of information in nats. In this paper, is an effort to establish a possible relation between geometry and information. For that, we start by investigating the relation of the Ricci scalar derived from a Robertson-Walker metric in the Friedmann cosmological model to the number of information bit $N$. We also investigate the relation of the Ricci scalar to entropy $S$. Finally, we use Landauer's principle of information and derive various new expressions for the Ricci scalar as a function of basic physical parameters of the universe. 


\section{The Robertson-Walker Metric and the Ricci Scalar}

In the context of general theory relativity, the standard Friedmann cosmological model can be described by the Robertson-Walker metric that has the following form [5]:

$$
d s^{2}=c^{2} d t^{2}-R^{2}(t)\left[\frac{d r^{2}}{1-k r^{2}}+r^{2}\left(d \theta^{2}+\sin ^{2} \theta d \phi^{2}\right)\right],
$$

where $k$ is a constant that can take the values of $-1,0,1$ giving three different kinds of spatial metric and $R(t)$ is the scale factor of the universe. In order to establish a relation between geometry of the universe and number of information in nats, let us look at the Ricci scalar $\mathscr{R}$ resulting from (2) which can be written as in $[5,6]$ :

$$
\mathscr{R}=g^{\mu \nu} R_{\mu \nu}=-\left(\frac{6 \ddot{R}}{c^{2} R}+\frac{6 \dot{R}^{2}}{c^{2} R^{2}}+\frac{6 k}{R^{2}}\right) .
$$

The scalar curvature is usually denoted by $\mathscr{R}$. It is defined as the trace of the Ricci curvature tensor with respect to the metric. Defining the Hubble constant $H(t)=\dot{R}(t) / R(t)$ and the deceleration parameter $q(t)=-\ddot{R}(t) R(t) / \dot{R}^{2}(t)$ [5] we obtain that $-\ddot{R} / R=q(t) H^{2}(t)$, also using relation $-k c^{2} / H^{2} R^{2}=1-\Omega$ [7], and (3) for the Ricci scalar can be written in the following way:

$$
\mathscr{R}=\frac{6 H^{2}}{c^{2}}(q(t)-\Omega(t))
$$

where $\Omega$ is the density parameter of the universe, and deceleration parameters can be in general functions of time $t$. $\Omega$ is defined as the ratio of the actual (or observed) density $\rho$ to the critical density $\rho_{c}$ of the Friedmann universe. The relation between the actual density and the critical density determines the overall geometry of the universe.

\section{Ricci Scalar and Its Relation to Entropy and the Number of Information in a Flat Universe}

In order to investigate the relation of the Ricci scalar to entropy and number of information $N$, we substitute (1) in (3), and we obtain that

$$
\mathscr{R}=\frac{6 \pi}{\ln 2} \frac{(q-\Omega)}{l_{P}^{2} N},
$$

where $\ell_{P}$ is the Planck length. Using the equation for the entropy given in Haranas and Gkigkitzis [3], namely

$$
S=N k_{B} \ln 2,
$$

where $N$ is the number of information in nats and $k_{B}$ is the Boltzmann constant we obtain that:

$$
\mathscr{R}=\frac{6 \pi k_{B}}{\ell_{p}^{2} S}(q-\Omega) .
$$

Therefore we find that the Ricci scalar is inversely proportional to the number of information $N$ or the entropy $S$, that is, $\mathscr{R} \propto 1 / N \propto 1 / S$. Furthermore, $q=\Omega((1+3 w) / 2)$, Kolb and Turner [8] an therefore the Ricci scalar can be also written as:

$$
\mathscr{R}=\frac{3 \pi k_{B} \Omega}{\ell_{P}^{2} S}(3 w-1),
$$

where $w=p / \rho$ is the equation of the state of a perfect fluid, $p$ is the pressure, and $\rho$ is the energy density. Similarly (7) can be written in the following way:

$$
\mathscr{R}=\frac{3 \pi \Omega}{\ln 2 l_{P}^{2} N}(3 w-1) \text {. }
$$

Next using (11) and the fact that $\Omega=\rho / \rho_{c}=8 \pi G \rho / 3 H^{2}$ [8] which is also equal to $\Omega=8 \pi G \rho \ln 2 \ell_{P}^{2} N / 3 \pi c^{2}$, after substituting in (11), we obtain that the Ricci scalar becomes equal to

$$
R=\frac{8 \pi G \rho}{c^{2}}(3 w-1),
$$

an expression that has units of $\mathrm{m}^{-2}$ and is independent of any of the four coordinates.

\section{Discussion and Numerical Results}

We examine the scalar curvature that constitutes a contraction of the Ricci tensor. Its meaning is very similar to the Gaussian curvature, and it tells how a surface area of a generalized sphere in curved space differs from the surface of a sphere in flat space. From (5), (7), and (9), we find that the Ricci scalar has an inversely proportional relation to number of information $N$ and entropy $S$. Thus we find that information and entropy can shape the universe geometry, by affecting its Ricci scalar. To use another interpretation we say that the Ricci scalar constitutes an information or entropy repository. Zero number of information would imply an infinite Ricci scalar. Therefore a nonzero number of information would imply a finite Ricci scalar, and therefore space time will unfold. Furthermore, the value of Ricci scalar linearly depends on the density parameter $\Omega$, and the $w$ is the equation of the state of a perfect fluid. The dependence of Ricci scalar on the $\Omega$ and $w$ will be examined in detail in a follow-up paper. In this letter we are mainly interested in establishing a relation between the Ricci scalar and the information number $N$ as well as entropy $S$. As a result of Landauer's information principle, in their recent paper, Faus and Fullana i Alfonso [9] postulate that "all physical systems of mass $M$ and energy $M c^{2}$ are equivalent to an amount of information in number of bits" of the order of

$$
N=\frac{M c^{2}}{\hbar H},
$$

where $M$ is the mass of the system, $c$ is the speed of light, $\hbar$ is Planck's constant, and $H$ is the Hubble constant. Assuming the universe to be the system of mass $M=M_{U}$ and using (5), 
we find that the Ricci scalar can be written in the following way:

$$
\mathscr{R}=\frac{6 \pi}{\ln 2}(q-\Omega) \frac{\hbar H}{\ell_{p}^{2} M c^{2}}=\frac{6 \pi}{\ln 2} \frac{(q-\Omega)}{\ell_{P}^{2}}\left(\frac{E_{\mathrm{gr}}}{E_{\mathrm{rel}}}\right),
$$

where $E_{\mathrm{gr}} \cong \hbar H$ is the gravitational energy [9] and $E_{\text {rel }}$ is the relativistic energy corresponding to the mass $M_{U}$. This is an expression for the Ricci scalar that depends on the ratio of the gravitational energy of the universe over its relativistic energy. Eliminating the mass of the universe using (15) in Haranas and Gkigkitzis [2] and also using the fact that $t_{P}^{2}=G \hbar / c^{5}$, we obtain an expression of the Ricci scalar in terms of fundamental parameters of physics to be

$$
\mathscr{R}=\frac{6 \pi}{\ln 2}(q-\Omega) \frac{\hbar H}{\ell_{p}^{2} M c^{2}}=\frac{6 \pi}{\ln 2}(q-\Omega) \frac{c H}{G M_{U}} .
$$

Eliminating the mass of the universe using (15) as in $[2,3]$, namely

$$
M=\frac{c^{3}}{G H},
$$

we further obtain that

$$
\mathscr{R}=\frac{6 \pi}{\ln 2}(q-\Omega) \frac{H^{2}}{c^{2}} .
$$

With reference to Islam [5] we can write that the cosmological constant lambda is equal to

$$
|\Lambda|=21 \frac{H_{0}^{2}}{c^{2}} .
$$

And therefore (15) can be written as

$$
\mathscr{R}=\frac{126 \pi}{\ln 2}(q-\Omega)|\Lambda| .
$$

Using (5) and the number of maximum number of information in the universe as it is given in Haranas and Gkigkitzis [3], namely, $N=4.661 \times 10^{122}$ nats, we obtain the following value for the Ricci scalar:

$$
\mathscr{R}=2.234 \times 10^{-52}(q-\Omega) \mathrm{m}^{-2} .
$$

Furthermore, using that the cosmological constant lambda is given by $\Lambda=1.117 \times 10^{-52} \mathrm{~m}^{-2}$ [10], (18) can be written as

$$
\mathscr{R}=2 \Lambda(q-\Omega) .
$$

This is a very small number that for any practical purpose can be taken to be approximately equal to zero.

\section{Conclusions}

In an effort to investigate the relation of the geometry of the universe to information, we have examined the Ricci scalar in the standard Robertson-Walker metric. Using a previously derived expression that relates the Hubble constant to the number of information $N$, we have expressed the Ricci scalar as a function of the number of information $N$. Next, using the relation that relates the entropy $S$ to the number of information, we have expressed the Ricci scalar in terms of the entropy $S$. Thus we find that information and entropy can shape the universe's geometry by affecting its Ricci scalar. The Ricci scalar scales in an inverse relation in both entropy and number of information and depend linearly on the difference of the deceleration and density parameters. Using a generalized expression for the number of information, we obtain alternative expressions for the Ricci scalar in terms of fundamental parameters of physics. Finally, we numerically evaluate the numerical value of the Ricci scalar for the maximum number of information $N$ that exists in the universe.

\section{Authors' Contribution}

Ioannis Haranas and Ioannis Gkigkitzis have contributed equally to the paper.

\section{Acknowledgments}

The authors want to thank an unknown reviewer who with his/her valuable comments help improved this paper.

\section{References}

[1] R. Landauer, "Dissipation and noise immunity in computation and communication," Nature, vol. 335 , no. 6193, pp. 779-784, 1988.

[2] I. Haranas and I. Gkigkitzis, "Bekestein bound of information number $N$ and its relation to cosmological parameters in a universe with and without cosmological constant," Modern Physics Letters A, vol. 28, no. 19, 2013.

[3] I. Haranas and I. Gkigkitzis, "The number of information bits related to the minimum quantum and gravitational masses in a vacuum dominated universe," Astrophysics and Space Science, vol. 346, no. 1, pp. 213-218, 2013.

[4] J. D. Bekenstein, "Universal upper bound on the entropy-toenergy ratio for bounded systems," Physical Review D, vol. 23, no. 2, pp. 287-298, 1981.

[5] J. N. Islam, An Introduction to Mathematical Cosmology, Cambridge University Press, New York, NY, USA, 1992.

[6] D. F. Lawden, Introduction to Tensor Calculus, Relativity and Cosmology, Dover Publications, New York, NY, USA, 2002.

[7] J. P. Ostriker, Development of Large-Scale Structure in the Universe, Accademia Nazionale dei Lincei Scuola Normale Superiore, PIZA, 1991.

[8] E. W. Kolb and M. S. Turner, The Early Universe, AddisonWesley, Reading, Mass, USA, 1990.

[9] A. A. Faus and M. J. Fullana i Alfonso, "Cosmic Background Bose Condensation (CBBC)," Astrophysics and Space Science, vol. 347, no. 1, pp. 193-196, 2013.

[10] L. M. Krauss and G. D. Starkman, "Life, the universe, and nothing: life and death in an ever-expanding universe," The Astrophysical Journal, vol. 531, no. 1, article 22, 1999. 

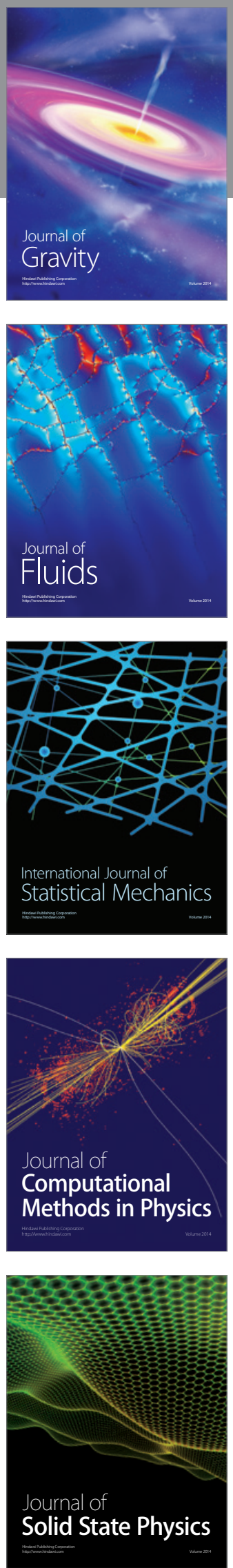

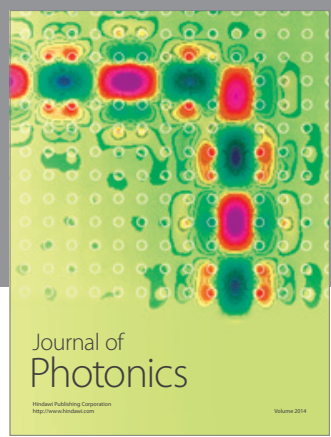

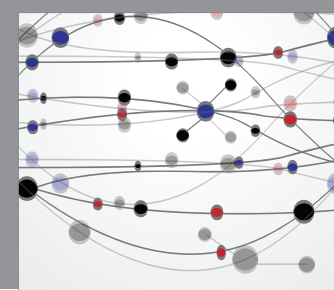

The Scientific World Journal

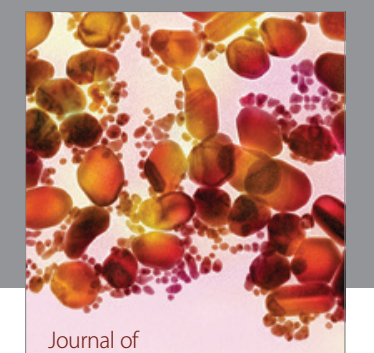

Soft Matter
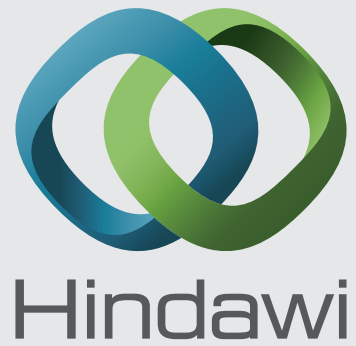

Submit your manuscripts at

http://www.hindawi.com
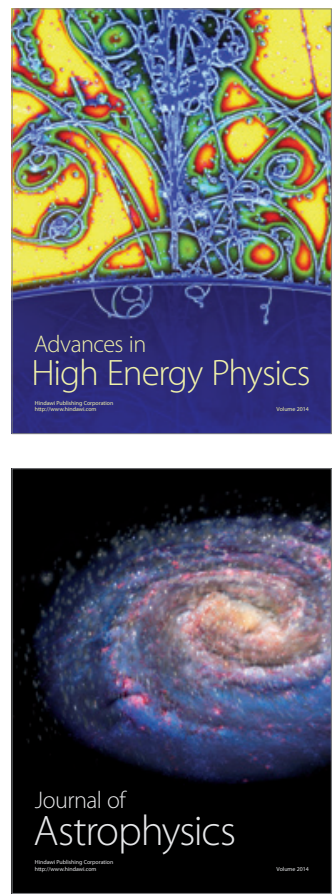
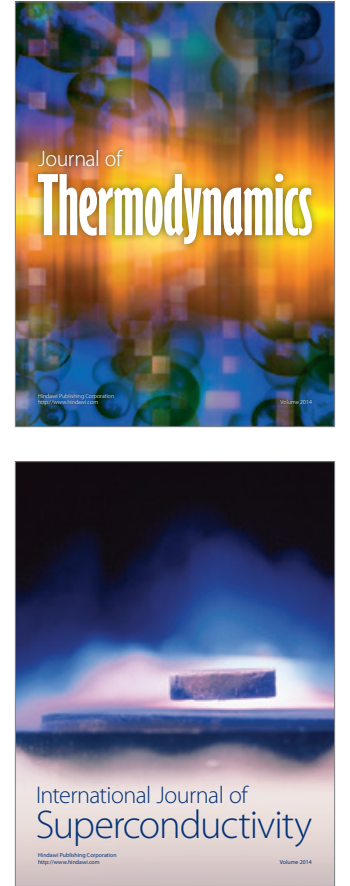
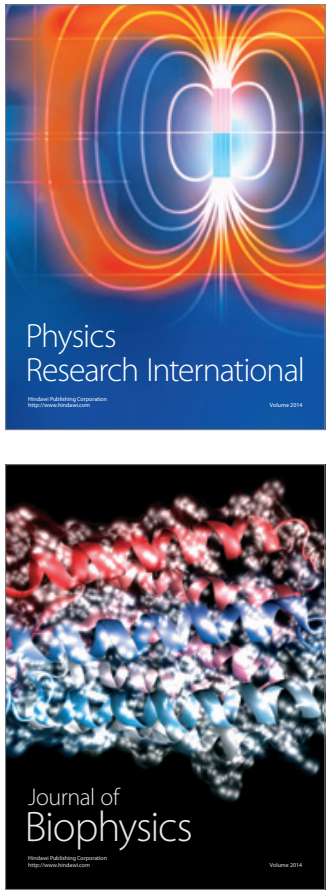
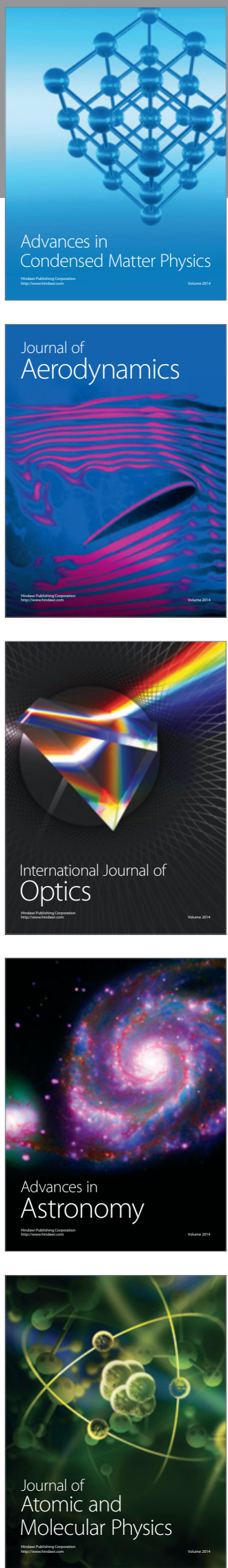